\title{
Magnetic interconnection of Saturn's polar regions: comparison of modelling results with Hubble Space Telescope UV auroral images
}

\author{
E. S. Belenkaya ${ }^{1}$, S. W. H. Cowley ${ }^{2}$, V. V. Kalegaev ${ }^{1}$, O. G. Barinov ${ }^{1}$, and W. O. Barinova ${ }^{1}$ \\ ${ }^{1}$ Lomonosov Moscow State University, Skobeltsyn Institute of Nuclear Physics, 1(2), Leninskie gory, GSP-1, Moscow \\ 119991, Russian Federation \\ ${ }^{2}$ Department of Physics \& Astronomy, University of Leicester, Leicester LE1 7RH, UK
}

Correspondence to: E. S. Belenkaya (elena@ dec1.sinp.msu.ru)

Received: 14 May 2013 - Revised: 15 July 2013 - Accepted: 16 July 2013 - Published: 28 August 2013

\begin{abstract}
We consider the magnetic interconnection of Saturn's northern and southern polar regions controlled by the interplanetary magnetic field (IMF), studying in particular the more complex and interesting case of southward IMF, when the Kronian magnetospheric magnetic field structure is the most twisted. The simpler case of northward IMF is also discussed. Knowledge of the magnetospheric magnetic field structure is very significant, for example, for investigation of the electric fields and field-aligned currents in Saturn's environment, particularly those which cause the auroral emissions. Here we modify the paraboloid magnetospheric magnetic field model employed in previous related studies by including higher multipole terms in Saturn's internal magnetic field, required for more detailed considerations of inter-hemispheric conjugacy, together with inclusion of a spheroidal boundary at the ionospheric level. The model is employed to map Southern Hemisphere auroral regions observed by the Hubble Space Telescope (HST) in 2008 under known IMF conditions to both the equatorial plane and the northern ionosphere. It is shown that the brightest auroral features map typically to the equatorial region between the central ring current and the outer magnetosphere, and that auroral features should be largely symmetric between the two hemispheres, except for a small poleward displacement and latitudinal narrowing in the Northern Hemisphere compared with the Southern Hemisphere due to the quadrupole field asymmetry. The latter features are in agreement with the conjugate auroras observed under near-equinoctial conditions in early 2009, when IMF data are not available.
\end{abstract}

Keywords. Magnetospheric physics (planetary magnetospheres)

\section{Introduction}

In this paper we study Saturn's ultraviolet (UV) auroral emissions observed by the Hubble Space Telescope (HST) in one hemisphere, and their mapping along field lines into the equatorial magnetosphere and the conjugate ionosphere. From Earth's orbit, it is usually only possible to observe auroras in one hemisphere, the southern auroras prior to the recent equinox in mid-August 2009, and the northern auroras afterwards. However, close to equinox, emissions from both hemispheres can be observed, if obliquely, with such images being obtained in the interval January-March 2009 (Nichols et al., 2009). It is assumed that Saturn's auroras are caused by accelerated magnetospheric electrons associated with strong upward-directed field-aligned currents. In order to better understand the origins of these currents and their location in the magnetosphere, bright auroral regions observed in HST images are mapped along field lines into Saturn's magnetosphere and the conjugate ionosphere using a newly refined version of the paraboloid magnetospheric magnetic field model that has been employed in previous related studies (e.g. Alexeev et al., 2006; Belenkaya et al., 2006, 2007, 2008). These studies show that the mapping depends strongly on the interplanetary magnetic field (IMF) penetrating the magnetosphere, with the most complex and interesting case occurring when the IMF is directed southward, in the same direction as the planet's equatorial field. Unfortunately no contemporaneous IMF data are available during the HST equinoctial campaign mentioned above, so that here we further study images of the southern aurora obtained in 2008 during intervals when the Cassini spacecraft was located just upstream of the near-noon bow shock (Belenkaya 
et al., 2010, 2011). The knowledge obtained can then be used to further consider the equinoctial images in future studies.

Southward IMF for Saturn is analogous to northward IMF for Earth, since the magnetic dipole axes are oppositely directed for these two planets. The interaction of the IMF with the terrestrial magnetosphere has been studied for a long time (e.g. Dungey, 1961; Cowley, 1973; Alexeev and Belenkaya, 1983; Belenkaya, 1998a, b; Blomberg et al., 2005). In particular, Figs. 1 and 2 of Blomberg et al. (2005), based on the results of Alexeev and Belenkaya (1983), illustrate the difference in the terrestrial magnetospheric magnetic field structure for southward and northward IMF using a simple spherical magnetic field model. As in the case of northward IMF for Earth, for southward IMF at Saturn two magnetic neutral points occur in the cusp regions, as also demonstrated by the paraboloid model under these conditions (e.g. Belenkaya et al., 2011). As the IMF at Saturn rotates from northward to southward, the two magnetic neutral points move from the low-latitude magnetopause to the cusp regions and are located inside the magnetosphere, leading to reconfiguration of the magnetic and electric field structure and convection, and modification of the interaction between the polar regions. Here we pay main attention to the latter point.

Kurth et al. (2009) noted that the processes generating Saturn's auroras are not well known yet. Gérard et al. (2004) showed that Saturn's auroral precipitation is usually stronger in the morning hours, and noted that the degree of development of the morning arc could be connected with the north-south orientation of the IMF. Cowley et al. (2004a, b) suggested that Saturn's auroral oval is caused by upwarddirected field-aligned currents occurring at the open-closed field line boundary, thus being strongly connected with the IMF, with the dawn-dusk asymmetry being associated with the effects of Dungey-cycle flow. The Kelvin-Helmholtz (KH) instability on the morning (mainly) and afternoon flanks of the magnetopause represents an alternative mechanism for auroral generation, as discussed, e.g., by Galopeau et al. (1995) in the context of Saturn kilometric radiation (SKR) emission. The latter authors noted in particular that the pre-noon SKR sources, which are caused by energetic electron precipitation, are magnetically conjugate in the Northern and Southern Hemispheres. In this scenario, brighter auroral emissions at dawn than at dusk are anticipated due to the enhanced flow shear at dawn between the magnetosheath and sub-corotating magnetospheric plasma. To clarify the nature of Saturn's bright UV emissions it is necessary to determine the places to which they are mapped in the equatorial magnetosphere (or/and magnetopause) for different IMF orientations. In the present paper we study this question.

\section{IMF dependence of the projection of Saturn's polar zones to the magnetospheric equatorial plane}

We begin by discussing the magnetic mapping that corresponds to Southern Hemisphere HST "image A" obtained at $\sim 21: 48$ UT on day 43 of 2008 under southward IMF conditions, studied previously by Belenkaya et al. (2011). The Kronian solar magnetospheric (KSM) $(X, Y, Z)$ field components measured by Cassini were $(0.20,0.85,0.24) \mathrm{nT}$, this vector representing a one-hour average taking account of the propagation delay between measurement at the spacecraft and arrival of effects in the ionosphere (see Belenkaya et al., 2010, for details). Here we use the same paraboloid magnetosphere field model as the latter authors (for details see Alexeev et al., 2006, and Belenkaya et al., 2006), but provide calculations of the mapping not only for the southern but also for the northern polar regions. The paraboloid model has been tested on several different planetary magnetospheres. It has provided good agreement with observations not only for the Earth's magnetosphere (e.g. Alexeev et al., 2001), but also at Saturn (Alexeev et al., 2006; Belenkaya et al., 2006, 2007, 2008, 2010, 2011), Jupiter (e.g. Alexeev and Belenkaya, 2005) and Mercury (Alexeev et al., 2008; Belenkaya et al., 2013). For the above case studied by Belenkaya et al. (2010), the model parameters chosen correspond to a high solar wind dynamic pressure $(\sim 0.1 \mathrm{nPa})$, with a distance from the planet's centre to the subsolar magnetopause of $R_{\mathrm{ss}}=17.5 R_{\mathrm{S}}$. The distances to the outer and inner edges of the ring current are taken to be $R_{\mathrm{rc} 1}=12.5 R_{\mathrm{S}}$ and $R_{\mathrm{rc} 2}=6.5 R_{\mathrm{S}}$, respectively, with the radial component of the ring current field just outside the thin current sheet at its outer edge being $B_{\mathrm{rc} 1}=3.62 \mathrm{nT}$. The distance to the inner edge of the tail current sheet is $R_{2}=14 R_{\mathrm{S}}$, with the field due to the tail current system at this distance, just outside the equatorial current sheet, being $B_{\mathrm{t}} / \alpha_{0}$, where $\alpha_{0}=$ $\left(1+2 R_{2} / R_{\mathrm{Ss}}\right)^{1 / 2}$ and $B_{\mathrm{t}}=8.7 \mathrm{nT}$. Here $R_{\mathrm{S}}$ is Saturn's 1 -bar equatorial radius equal to $60268 \mathrm{~km}$. The coefficient of IMF penetration appropriate to Saturn, $k_{\mathrm{S}}$, is not known with certainty, but is taken to be equal to 0.2 , corresponding to values determined directly at Earth (e.g. Cowley and Hughes, 1983; Petrukovich, 2011). Field lines are computed to a maximum distance of $2000 R_{\mathrm{S}}$ down-tail, experimentation showing that the area of the open field region hardly changes if larger values are employed. Cowley et al. (2004a, b) estimated the length of Saturn's tail to be $\sim 1500 R_{\mathrm{S}}$, consistent with this choice. The corresponding boundary between open and closed field lines was then shown by Belenkaya et al. (2011) to be in reasonable agreement with the poleward boundary of auroral emissions observed in image A.

In Fig. 1 we show field mappings to the KSM equatorial plane, bounded by the parabolic magnetopause shown by the outer black line, viewed from the north with the Sun to the right. Panel (a) shows a large-scale view extending beyond $\sim 500 R_{\mathrm{S}}$ down-tail, while panel (b) shows a closer view of the near-planet region. The internal black curve across the 


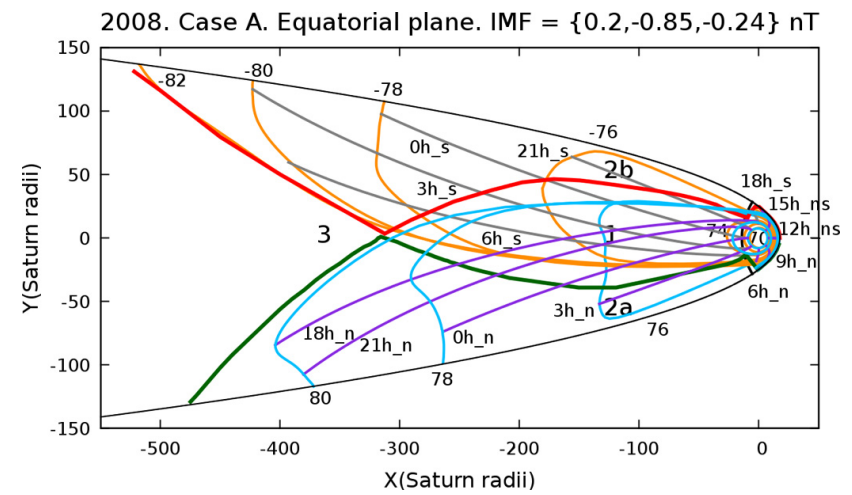

2008. Case A. Equatorial plane close to Saturn. IMF $=\{0.2,-0.85,-0.24\} n T$

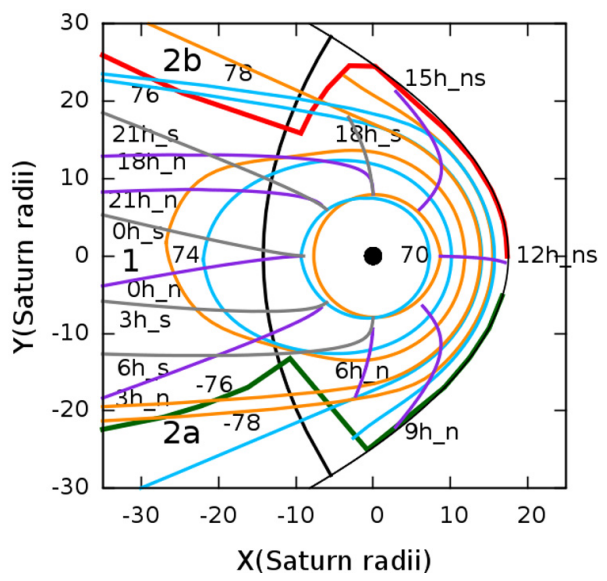

Fig. 1. Projections of polar regions into Saturn's equatorial plane for conditions corresponding to HST image A of Belenkaya et al. (2011), in which the KSM IMF vector has $(X, Y, Z)$ components of $(0.20,-0.85,-0.24) \mathrm{nT}$. Panel (a) shows an extended view, while panel (b) shows a view of the nearer-planet region. The green and red lines mark the boundary between open and closed field lines mapped from the north and south polar regions, respectively. Region " 1 " between the red and green lines for $x>-312 R_{\mathrm{S}}$ thus corresponds to the region of closed field lines, while region " 3 " between these lines for $x<-312 R_{\mathrm{S}}$ contains interplanetary field lines that pass through the tail. Regions " $2 a$ " and " $2 \mathrm{~b}$ " correspond to open field lines mapping to the northern and southern polar caps, respectively. Projections of constant latitude circles from the north and south are shown by the blue and orange lines, respectively, marked with the corresponding latitudes at $2^{\circ}$ intervals. Purple and grey curves show corresponding northern and southern lines of constant MLT at $3 \mathrm{~h}$ intervals, as marked, where " $\mathrm{n}$ " and " $\mathrm{s}$ " correspond to "north" and "south" respectively.

tail marks the inner edge of the tail current sheet, while the two black circles in panel (b) mark the inner and outer edges of the model ring current. The equatorial plane is divided into four regions by the green and red lines, mapping to the openclosed field line boundaries in the Northern and Southern Hemispheres, respectively. Region " 1 " corresponds to closed field lines, "2a" to open field lines mapping to the north polar cap, " $2 \mathrm{~b}$ " to open field lines mapping to the south polar

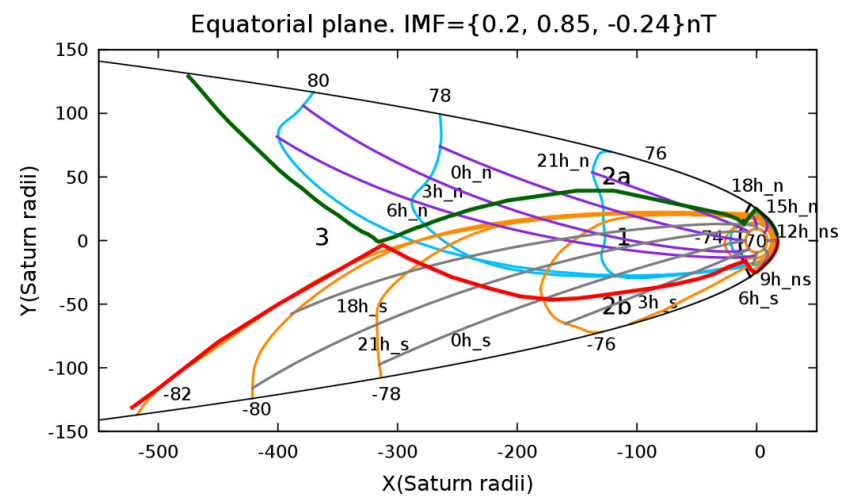

Equatorial plane near Saturn.IMF $=\{0.2,0.85,-0.24\} \mathrm{nT}$

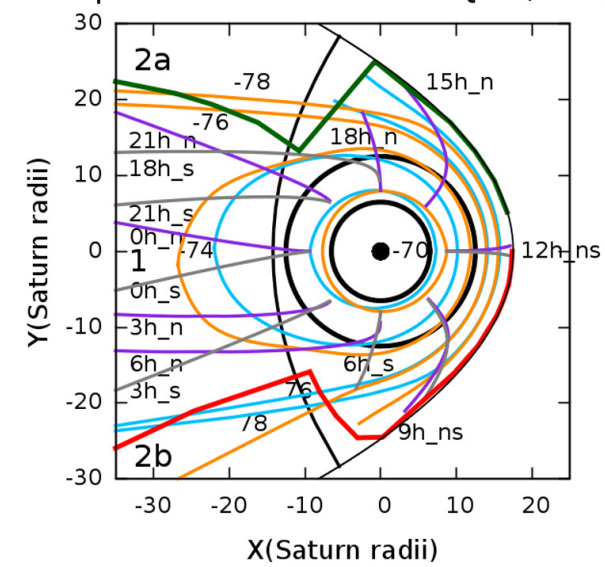

Fig. 2. As in Fig. 1 but for IMF vector $(0.20,0.85,-0.24) n T$.

cap, and " 3 " to interplanetary lines that thread the tail. The blue and orange curves then show the mapping of constant latitude circles from the northern and southern polar ionospheres, respectively, from $\pm 70^{\circ}$ to $\pm 82^{\circ}$, at steps of $2^{\circ}$. For $\pm 70^{\circ}$ to $\pm 74^{\circ}$ the curves for the two hemispheres almost coincide, while for $\pm 76^{\circ}$ and above they are significantly different from each other. The lines starting from $+82^{\circ}$ do not intersect the equatorial plane at all, while the lines from $-82^{\circ}$ in the MLT range from $\sim 8$ to $11 \mathrm{~h}$ do pass through the equatorial plane as shown. Similarly, the purple and grey lines show mappings of lines of constant MLT from the Northern and Southern Hemispheres, respectively, at steps of $3 \mathrm{~h}$.

A separatrix field line connecting the northern and southern magnetic nulls intersects the equatorial plane where the green and red curves meet. A potential drop equal to the potential drop between the two magnetic nulls is applied to the "ends" of the separatrices. So, along a separatrix strong fieldaligned currents flow. For the case studied, at the southern polar cap these currents are associated with the dawn, while at the northern polar cap with the dusk.

As noted above, the bright auroras at Saturn are most often seen in the dawn sector in both hemispheres, when they can be observed simultaneously (e.g. Nichols et al., 2009). It can be seen in Fig. 1 that the dawn part of the northern polar 
Equatorial plane. IMF $=\{0.2,-0.85,0.24\} \mathrm{nT}$

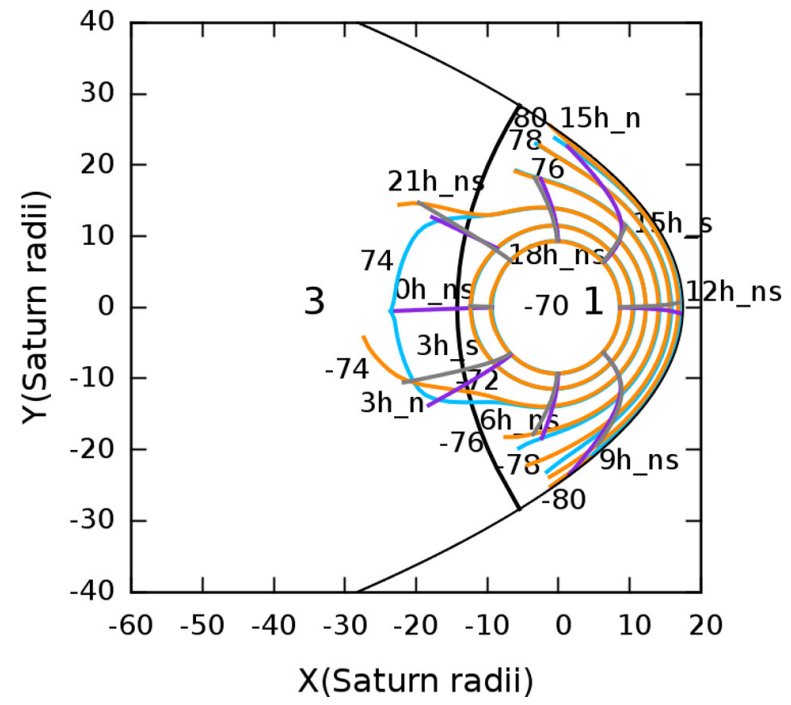

Fig. 3. As in Fig. 1 but for $\operatorname{IMF}$ vector $(0.20,-0.85,0.24) n T$.

region is projected to the dawn and central equatorial magnetosphere to down-tail distances up to $-400 R_{\mathrm{E}}$ in this case, while the dawn part of the southern polar region is projected closer to the $x$ axis of the KSM system. The dawn-noon and dusk-noon flanks for positive $x$ (Fig. 1b) are connected with the high-latitude closed southern and northern regions, as both the blue (northern) and orange (southern) curves projections of constant latitude circles - are located there. It is then of interest to consider the corresponding case for southward IMF and positive $B_{y}$. In Fig. 2 we thus show projections to the equatorial magnetospheric plane for the IMF vector $(0.20,0.85,-0.24) \mathrm{nT}$, in the same format as Fig. 1, in which the field lines are mirror images in the noon-midnight plane. Now the dawn part of the southern polar region is mapped to the dawn equatorial flank of the magnetosphere up to $-500 R_{\mathrm{E}}$, while the dawn portion of the northern polar cap is projected closer to the $x$ axis of the KSM. In Fig. $2 \mathrm{~b}$ it is seen that the dawn-noon and dusk-noon flanks for positive $x$ are connected with the high-latitude closed field line regions of both polar caps. Thus, for southward IMF, the flanks of the magnetosphere with $x>0$ are connected with both highlatitude closed regions independent on the sign of $B_{y}$ IMF. This fact could be taken into account if one would like to explain the dawn polar emissions for southward IMF by the velocity shift between the solar wind flow and the co-rotating magnetospheric plasma flow on the dawn magnetopause.

Figure 3 shows how the projections are modified for northward IMF, specifically for the IMF vector $(0.20,-0.85$, $0.24) \mathrm{nT}$, using the same line formats as in Figs. 1 and 2. Only the constant-latitude curves for $\pm 74^{\circ}$ are slightly distinct from each other, while the others almost coincide. Similar comments apply to the constant MLT lines; only the curves for $3 \mathrm{~h} \mathrm{MLT}$ are distinct from each other in this case, while the others almost coincide. In this case the pre-noon (postnoon) sectors of the high-latitude closed field line regions of both hemispheres are mapped to the dawn (dusk) flank of the Kronian magnetosphere, as is usually expected.

\section{Model calculations for the multipole planetary field and spheroidal ionosphere}

Irrespective of the origin of Saturn's auroras, whether velocity shears, plasma injections, KH waves as discussed above (see also, e.g., Masters et al., 2012), or ultra-low frequency (ULF) waves in the outer magnetosphere (Meredith et al., 2013), it is evidently important to map accurately between the planet's polar regions and the equatorial plane, and between conjugate ionospheres. In order to do this we have newly augmented the paraboloid model employed previously to include not only the internal planetary dipole field, but also the well-determined axial quadrupole and octupole components, the quadrupole term now removing north-south symmetry. We also take account of the distinctly flattened figure of the planet, where the ionospheric layer of maximum auroral luminosity is taken to be a spheroid of revolution about the spin axis. In this section we describe and discuss the modified model, while in Sect. 4 we apply it to further investigation of the HST images analysed by Belenkaya et al. (2011).

The magnetic field inside the Kronian magnetosphere is represented as a sum of fields produced by currents flowing in Saturn's core $\boldsymbol{B}_{\text {int }}$ (internal), and external fields produced by magnetospheric current systems $\boldsymbol{B}_{\text {ext }}$ (the latter field being described in detail by Alexeev et al., 2006, and Belenkaya et al., 2006):

$\boldsymbol{B}=\boldsymbol{B}_{\text {int }}+\boldsymbol{B}_{\text {ext }}$.

The internal field outside the planet can be described as the gradient of a scalar potential represented as a series of spherical harmonics:

$\boldsymbol{B}_{\text {int }}=-\nabla \Phi$,

where

$$
\begin{aligned}
\Phi & =R_{\mathrm{S}}\left(g_{10} \cos \theta\left(\frac{R_{\mathrm{S}}}{r}\right)^{2}\right. \\
& +\frac{1}{2} g_{20}\left(3 \cos ^{2} \theta-1\right)\left(\frac{R_{\mathrm{S}}}{r}\right)^{3} \\
& \left.+\frac{1}{2} g_{30} \cos \theta\left(5 \cos ^{2} \theta-3\right)\left(\frac{R_{\mathrm{S}}}{r}\right)^{4}\right) .
\end{aligned}
$$

The internal magnetic field components in spherical coordinates can then be written as

$$
\begin{aligned}
B_{\text {int } r} & =2 g_{10} \cos \theta\left(\frac{R_{\mathrm{S}}}{r}\right)^{3}+\frac{3}{2} g_{20}\left(3 \cos ^{2} \theta-1\right)\left(\frac{R_{\mathrm{S}}}{r}\right)^{4} \\
& +2 g_{30} \cos \theta\left(5 \cos ^{2} \theta-3\right)\left(\frac{R_{\mathrm{S}}}{r}\right)^{5},
\end{aligned}
$$



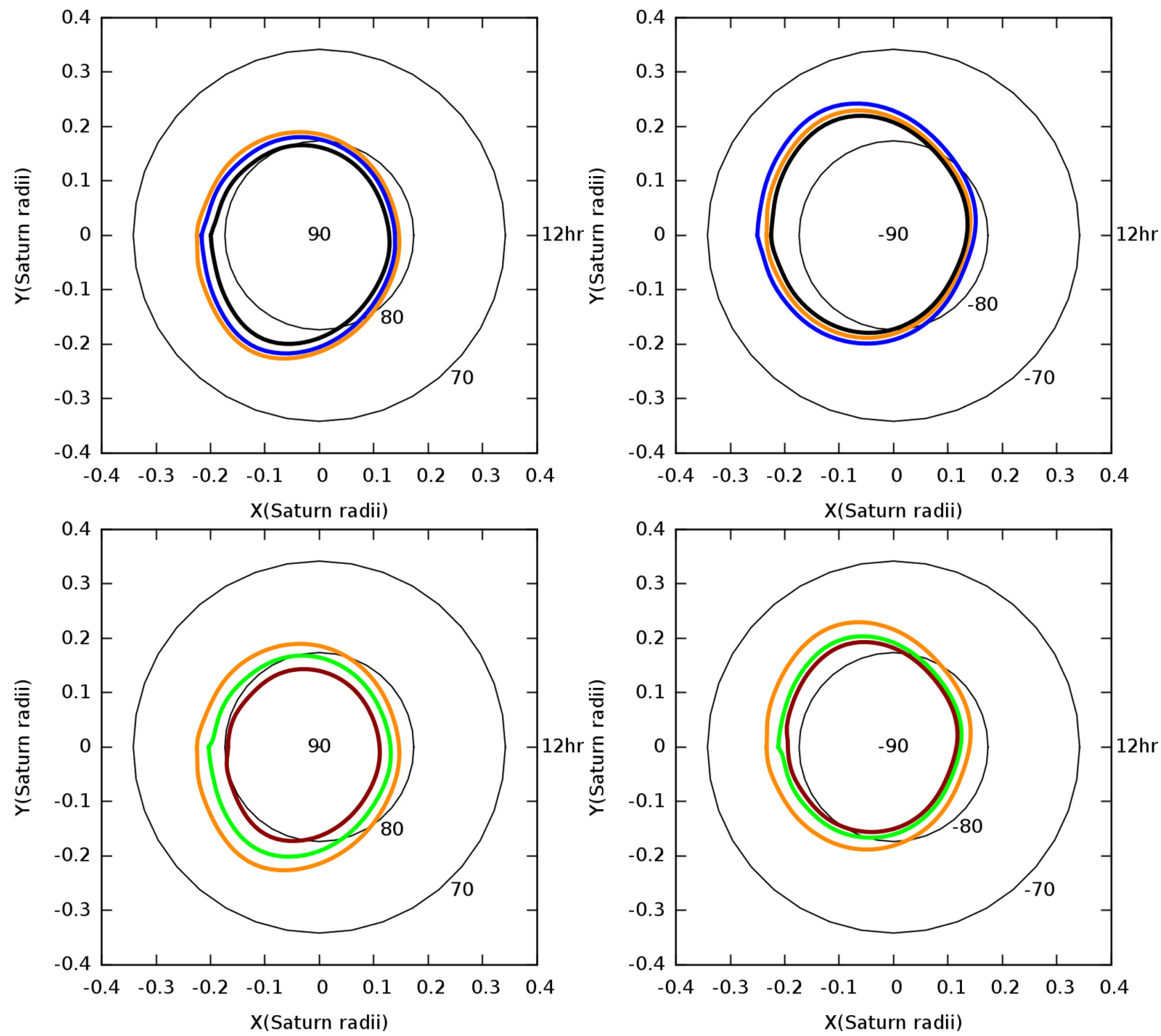

Fig. 4. Open field regions in Saturn's northern (left) and southern (right) polar caps for various field and ionospheric surface models. (a) Orange (outer) oval marks the open flux boundary for the dipole field model and a spherical ionosphere. Blue (intermediate) oval marks the open flux boundary for the dipole plus quadrupole field and a spherical ionosphere. Black (inner) oval is for the open flux boundary for the multipole (dipole, quadrupole, octupole) field model and a spherical ionosphere. (b) The same for the southern polar cap except that now the oval for the dipole field and the spherical ionosphere (orange) is intermediate, the dipole plus quadrupole for the spherical ionosphere (blue) is the outer oval, and the multipole with spherical ionosphere (black) oval is the inner. (c) For northward hemisphere the orange outer oval marks the open flux boundary for the dipole field and spherical ionosphere model, while the green intermediate oval marks the open flux boundary for the dipole field and a spheroidal ionosphere, and the brown inner oval is the open flux boundary for the multipole (dipole, quadrupole, octupole) field and spheroidal ionosphere model. (d) The same for the southern polar cap.

$$
\begin{aligned}
B_{\text {int } \theta} & =g_{10} \sin \theta\left(\frac{R_{\mathrm{S}}}{r}\right)^{3}+3 g_{20} \sin \theta \cos \theta\left(\frac{R_{\mathrm{S}}}{r}\right)^{4} \\
& +\frac{3}{2} g_{30} \sin \theta\left(5 \cos ^{2} \theta-1\right)\left(\frac{R_{\mathrm{S}}}{r}\right)^{5},
\end{aligned}
$$

$B_{\text {int } \varphi}=0$.
As indicated by these equations, the most recent determination of the internal field of Saturn by Burton et al. (2010), obtained from Cassini field measurements inside the orbit of Enceladus $\left(r<3.95 R_{\mathrm{S}}\right)$, includes three non-zero terms corresponding to axial dipole, quadrupole, and octupole components. This gives an axially symmetric field with no azimuthal component, but with a north-south asymmetry corresponding approximately to a small displacement of the dipole 
centre along the axis into the Northern Hemisphere. The three coefficients are $21136\left(g_{10}\right), 1526\left(g_{20}\right)$, and $2219 \mathrm{nT}$ $\left(g_{30}\right)$ (Table 1 of Burton et al., 2010). Following the earlier discussion of Cowley and Bunce (2003), here we also take the spheroidal ionosphere to be an ellipse of revolution located at a height of $1100 \mathrm{~km}$ above the 1-bar surface (Gérard et al., 2009). Following the IAU convention for the latter surface, the equatorial and polar radii of the ionosphere are thus taken to be $R_{\mathrm{e}}=61368 \mathrm{~km}=1.0182 R_{\mathrm{S}}$ and $R_{\mathrm{p}}=55464 \mathrm{~km}=0.9203 R_{\mathrm{S}}$, respectively, as presented in the paper by Radioti et al. (2011).

We now use the modified model to calculate the open field line regions for conditions corresponding to HST image A, as discussed above in relation to Fig. 1. Results are shown in Fig. 4 for the northern (left) and southern (right) polar caps, respectively. In the top panel the open field area is shown for the spherical ionosphere and for the dipole field (orange oval), for the dipole plus quadrupole magnetic field (blue oval), and for the dipole, quadrupole and octupole terms (black oval). In Fig. 4a it is seen that in the Northern Hemisphere the quadrupole term decreases the open field line area relative to the case of a dipole field (the blue oval lies inside the orange oval), while in the Southern Hemisphere (Fig. 4b) the situation is opposite, with the quadrupole increasing the open flux area relative to the dipole term (the blue oval lies outside the orange oval). This is due to the quadrupole term increasing the field strength compared with the dipole in the Northern Hemisphere, while decreasing it in the Southern Hemisphere, approximately equivalent to a northward axial shift of the dipole centre. In the Northern Hemisphere (Fig. 4a) the angular radius of the open field line region decreases by $\sim 0.5^{\circ}$ due to the quadrupole term, while in the Southern Hemisphere (Fig. 4b) it increases by $\sim 0.8^{\circ}$. Related behaviour was found for Mercury by Alexeev et al. (2008), and its effect on the Hermean polar caps was described by Belenkaya et al. (2013). The octupole term decreases the open flux area in both polar caps (black oval is inside all the others in Figs. 4a, b), since it increases the field strength symmetrically in both hemispheres.

In the lower panels of Fig. 4 the open flux in the Northern (Fig. 4c) and Southern (Fig. 4d) Hemispheres is shown for the dipole field and the spherical ionospheric model (orange oval), for the dipole field and the spheroidal ionospheric model (green oval), and for the multipole (dipole, quadrupole, and octupole) internal field and the spheroidal model (brown oval). It is seen from both Figs. $4 \mathrm{c}$ and $4 \mathrm{~d}$ that the spheroidal form decreases the open flux radius in both hemispheres by $\sim 1.5^{\circ}$ (the green oval is inside the orange oval), and that the multipole terms (quadrupole plus octupole) additionally decrease it by $\sim 1.5^{\circ}$ for the northern polar cap (Fig. $4 \mathrm{c}$ ) and by $\sim 0.5^{\circ}$ for the southern polar cap (Fig. 4d). Thus, in both polar caps the brown oval marking the open flux boundary for the multipole field and spheroidal ionosphere is located inside the ovals for the dipole field with different forms for the ionospheric level. The total decrease of the angular radius of the boundary is $\sim 3^{\circ}$ in the Northern Hemisphere (Fig. 4c) and 2-2.5 in the Southern Hemisphere, where the decrease is bigger on the nightside than on the dayside (Fig. 4d).

The dawn-dusk asymmetry in the open field regions in Fig. 4 is due to the IMF $B_{y}$ field component. The angular radius of the northern open field region in the noon-midnight direction is $\sim 8.2^{\circ}$, while in the dawn-dusk direction it is $\sim 8.8^{\circ}$ (Fig. $4 \mathrm{c}$ ). In the Southern Hemisphere the angular radius of the open flux in the noon-midnight direction is $\sim 9.1^{\circ}$ while in the dawn-dusk direction it is $\sim 10^{\circ}$ (Fig. 4d). Thus the average radius of the southern open flux region is bigger than the northern radius by $\sim 1^{\circ}$. Nichols et al. (2009), studying the first equinoctial auroras observed simultaneously in both hemispheres, found that the southern oval is $\sim 1.5^{\circ} \mathrm{big}$ ger than the northern one, which is in good accord with our result.

\section{Mapping bright southern auroral features to the equatorial plane and to the Northern Hemisphere using the augmented model}

HST southern auroral image A obtained on day 43 of 2008 is shown in Fig. 5 (see also the top panel of Fig. 2 of Belenkaya et al., 2011). Dotted circles and radial lines show lines of constant latitude and longitude, respectively, at $10^{\circ}$ steps, while red crosses mark the boundaries of the oval. Here we highlight in green three bright regions in the image, specifically where the brightness lies within $16 \%$ of a local maximum in UV intensity in the image, corresponding to a large dawn auroral patch, a narrow dusk arc aligned in the noon-midnight direction, and a small bright spot near noon. At $6 \mathrm{~h}$ MLT the bright patch is located between -74.7 and $-81.3^{\circ}$, while near $10 \mathrm{~h}$ MLT it is located between -73.7 and $-76.3^{\circ}$. The orange and brown ovals in Fig. 5 correspond to the same ovals as in Fig. 4d, marking the open field boundaries for the dipole plus spherical ionosphere model, and the multipole plus spheroidal ionosphere model, respectively. It is seen that the latter model leads to better coincidence between the calculated open flux boundary and the poleward boundary of the aurora.

Figure 6 shows the southern polar region in the same format as the panels of Fig. 4, where the boundaries of the bright auroral regions in image A in Fig. 5 have been marked with symbols corresponding to the starting points of mapped field lines that will be discussed below. The poleward and equatorward boundaries of the dawn patch are marked by green squares and triangles at half-hour intervals of MLT, the boundaries of the dusk arc are similarly indicated by brown squares and purple triangles, while the near-noon spot is indicated by the red circle. The former points are numbered for ease of identification of the mapped field lines in subsequent figures. The brown oval marks the boundary of open 


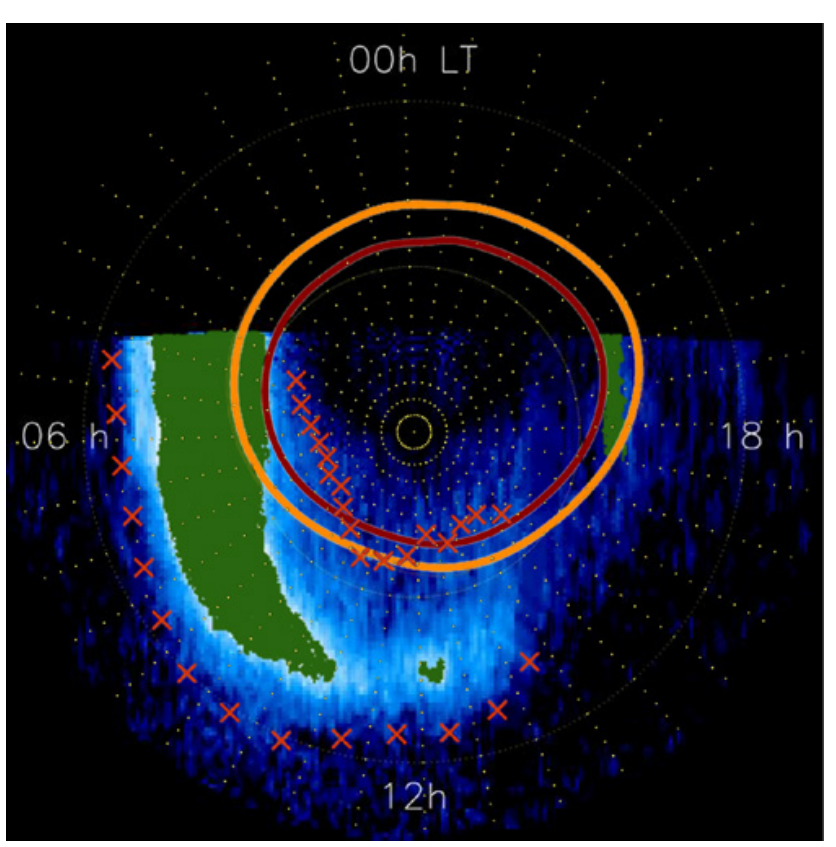

Fig. 5. Plot showing HST UV image A of Saturn's southern aurora, projected onto a spheroidal surface $1100 \mathrm{~km}$ above the atmospheric 1-bar level, with noon at the bottom and dawn to the left. The view is thus 'through' the planet from the north. Dotted lines show a latitude-longitude grid at $10^{\circ}$ intervals. The locations of the poleward and equatorward boundaries of the emission are shown by red crosses, determined at $10^{\circ}$ intervals of longitude from a $5 \mathrm{kR}$ limiting emission intensity. The superposed solid orange curve shows the open field region for $k_{\mathrm{S}}=0.2$, dipole planetary field and spherical ionosphere. The brown curve marks the open field region for the multipole magnetic field and spheroidal ionosphere. Green areas mark regions of bright emission (a large dawn auroral patch, a narrow dusk arc aligned in the noon-midnight direction, and a small bright spot near noon) with brightness higher that $16 \%$ of the local maximum brightness in each region.

field lines for the multipole field and spheroidal ionosphere model, as shown in Fig. 5.

Figure 7 shows the projection of these points along field lines into the equatorial plane shown previously in Fig. 1, using the same symbol types and numbers. Panel (a) again shows an overall view, while panel (b) shows a close-up of the near-planet region. It can be seen that the equatorward boundary of the dawn patch (green triangles) maps into the dawn-noon sector of the ring current region, while the poleward boundary maps close to the boundary of the open field region. Part of the poleward boundary is located on open field lines (green squares numbered 2 to 5), which maps close to the boundary of the southern open field region $2 b$, shown by the red line in Fig. 7a. Green square 1 is on closed field lines mapping close to this boundary near midnight, while green squares 6 to 15 are also on closed field lines, now mapping close to the boundary with open field lines from the northern polar cap (region 2a), shown by the green line in Fig. 7a. With

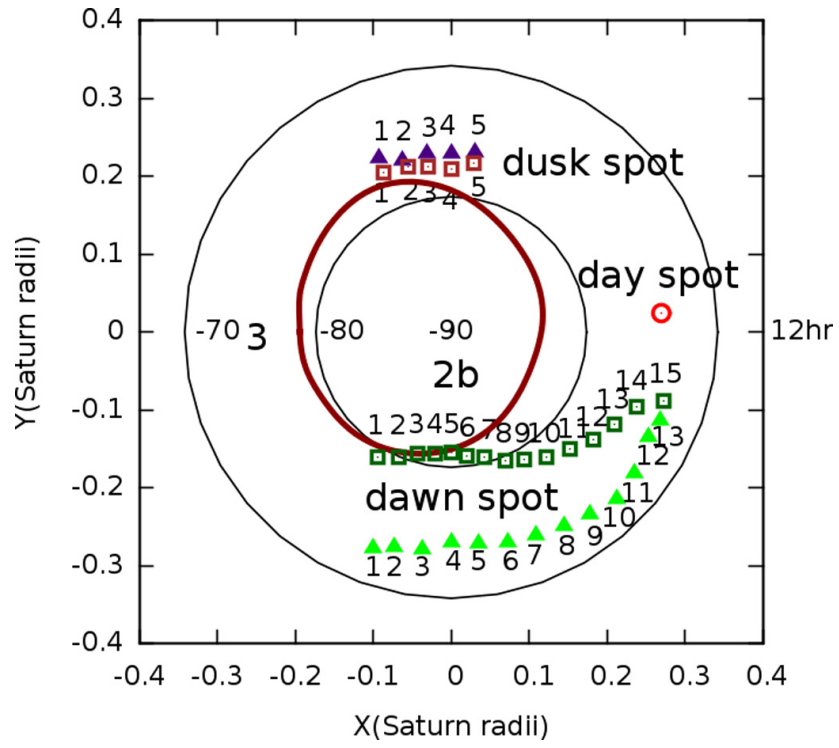

Fig. 6. Southern polar region for image $A$ in the same format as the panels of Fig. 4, with the boundaries of the bright auroral regions shown by the green regions in Fig. 5 marked at half-hour intervals of MLT by numbered symbols. The brown oval shows the boundary between open and closed field lines for the multipole field and spheroidal ionosphere model, as in Fig. 5.

increasing number along the latter sequence, the points move towards noon and into the ring current region, where they meet the mapped points from the equatorward dawn patch boundary. Green squares with numbers 8 to 15 are located in the pre-noon magnetospheric flank with $x>0$ and $y<0$. It can also be seen in Fig. 7b that the near-noon spot (red circle) maps inside the noon ring current. The dusk arc (brown squares and purple triangles) is located on closed field lines between the dusk boundary of open flux from the southern polar cap shown by the red line, and the outer edge of the dusk-side ring current.

Although we have no observations of the northern emissions corresponding to southern image $\mathrm{A}$, it is of interest to examine the predictions of the model obtained by mapping the above auroral features into the northern ionosphere. These are shown in Fig. 8 using the same symbol types and numbers, and where the brown oval shows the region of open field lines determined from the multipole field plus spheroidal ionosphere model (the same as the brown oval in Fig. 4c). Comparison of Figs. 6 and 8 shows overall that corresponding points map to higher latitudes in the northern than in the Southern Hemisphere, due to the quadrupole asymmetry in the internal field. It can also been seen that for the equatorward boundary of the dawn patch (green triangles), the MLT shift of the footprints is not significant. The same is true of the large-numbered points of the poleward boundary (green squares), but as these points approach the open-closed boundary they become strongly displaced around it, to midnight for point 7 , and to dusk for points 6 and 1, due to these 


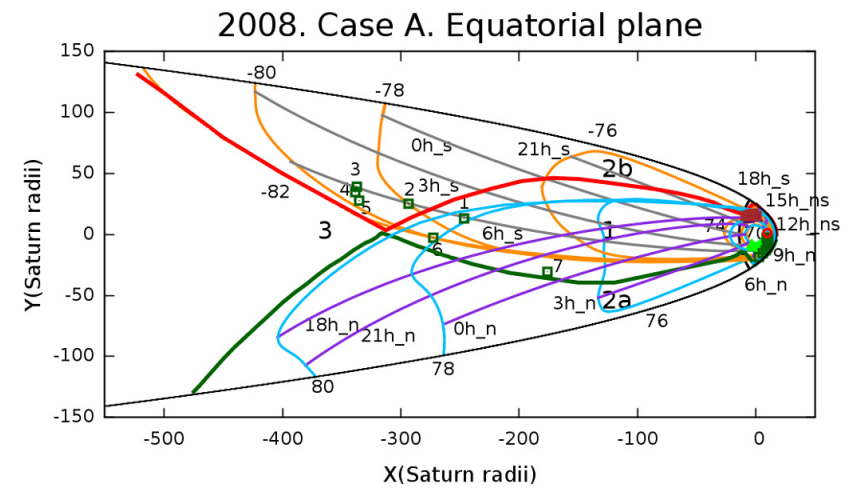

2008-Case A. Spot projections. IMF $=\{0.2,-0.85,-0.24\} \mathrm{nT}$

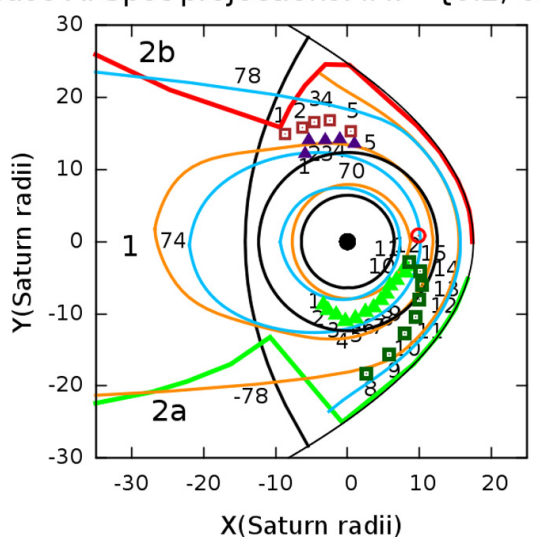

Fig. 7. As in Fig. 1 but with the boundary points of the bright auroral regions (a large dawn auroral patch, a narrow dusk arc aligned in the noon-midnight direction, and a small bright spot near noon) mapped into the equatorial plane superposed.

lines passing close to the magnetic null points. Of course, points $2-5$, located on southern open field lines, do not map into the northern ionosphere at all. Overall, the dawn patch retains a similar shape in the Northern Hemisphere, but is narrower. The mappings of the near-noon spot and dusk arc follow related expectations.

Consistent with the previous conclusions of Belenkaya et al. (2011), these mapping results indicate that the poleward part of the dawn patch emissions in image A could be generated by velocity shears at the boundaries of the open flux region and the dawn-noon equatorial magnetospheric flank. However, the equatorward parts of these emissions as well as the near-noon spot are likely associated with fieldaligned currents generated in the dawn-noon sector of the ring current. Modulation of the field-aligned currents in the latter region by ULF waves, driven, for example, by the driftbounce resonance mechanism, can then lead to drifting auroral patches in this region, as recently discussed by Meredith et al. (2013).

For comparison with these results for image A obtained under southward IMF conditions, we now also consider related results for "image C" of Belenkaya et al. (2011), ob-

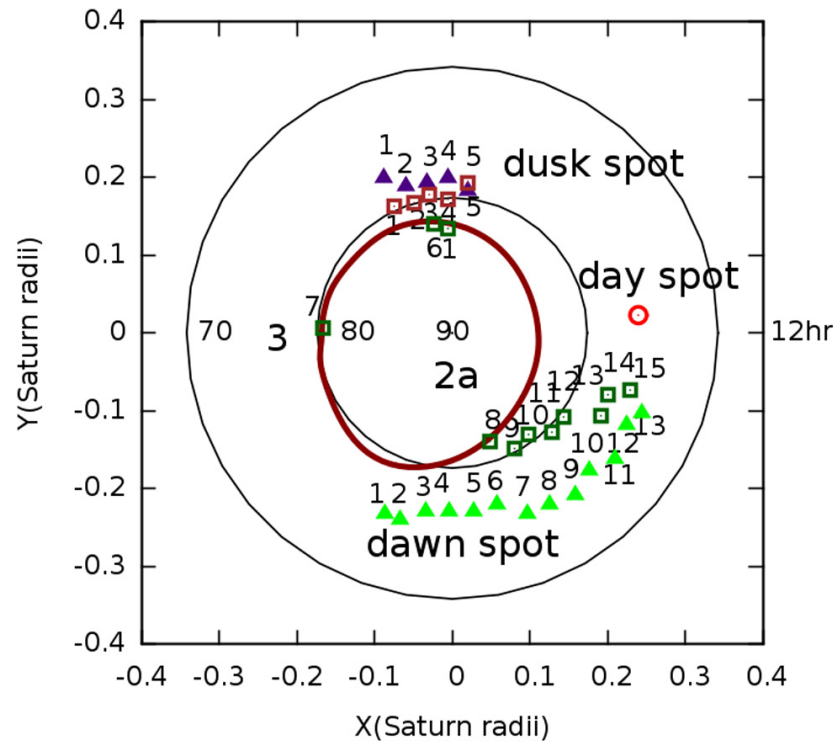

Fig. 8. Northern polar region corresponding to image A, showing the auroral boundary points plotted in Fig. 6 mapped along field lines into the northern ionosphere. The brown oval shows the boundary between open and closed field lines for the multipole field and spheroidal ionosphere model.

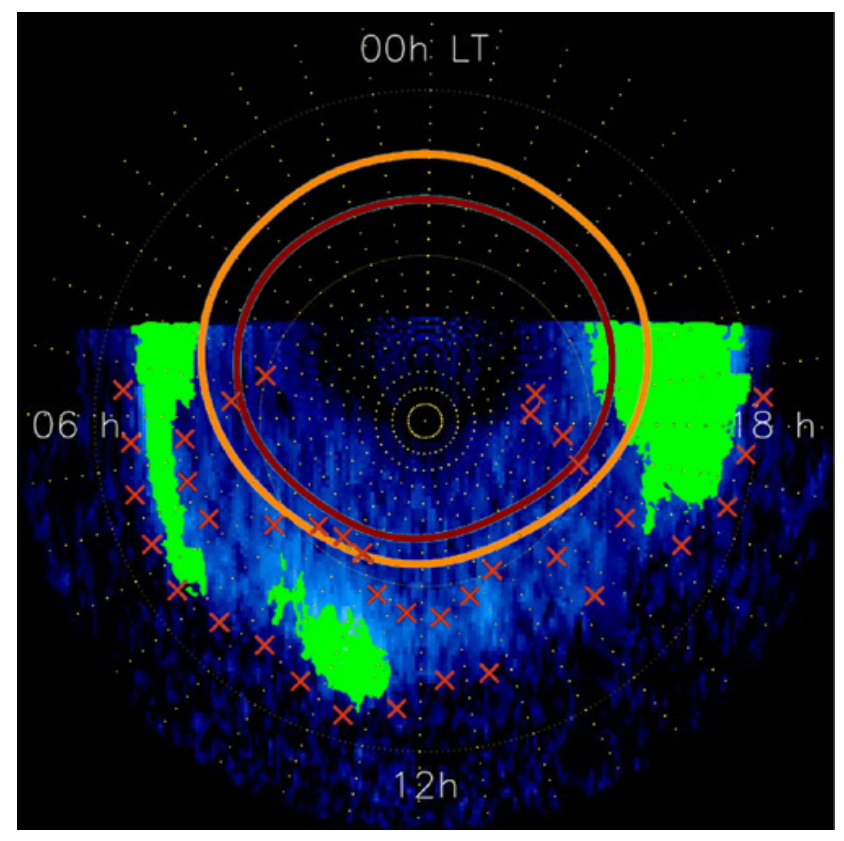

Fig. 9. HST UV southern auroral image $C$ obtained at $\sim 07: 10$ UT on day 45 of 2008 shown in the same format as Fig. 5. Bright emission regions now are a dawn arc, near-noon spot, and a dusk patch.

tained at $\sim$ 07:10 UT on day 45 of 2008 under northward IMF conditions, specifically for KSM IMF vector $(-0.11$, $0.28,0.25) \mathrm{nT}$. Figure 9 shows the UV image in the same format as Fig. 5, where the orange and brown ovals again mark the boundary of open field lines in the Southern Hemisphere 


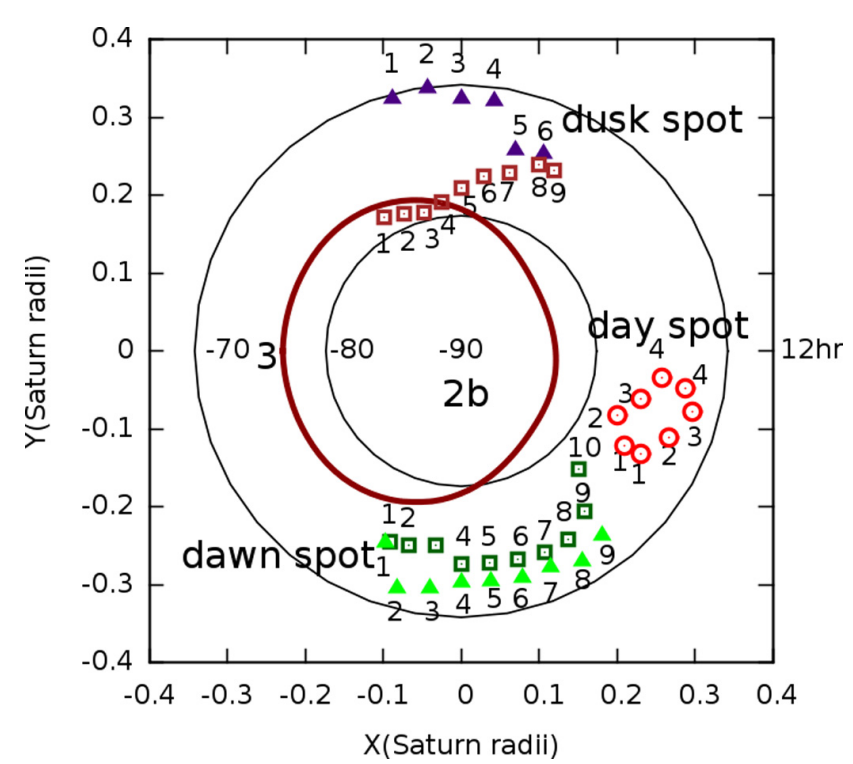

Fig. 10. Southern auroral boundaries for image C, shown in the same format as Fig. 6.

2008. Case C. Spot projections. IMF $=\left\{\begin{array}{lll}-0.11 & 0.28 & 0.25\end{array}\right\}$

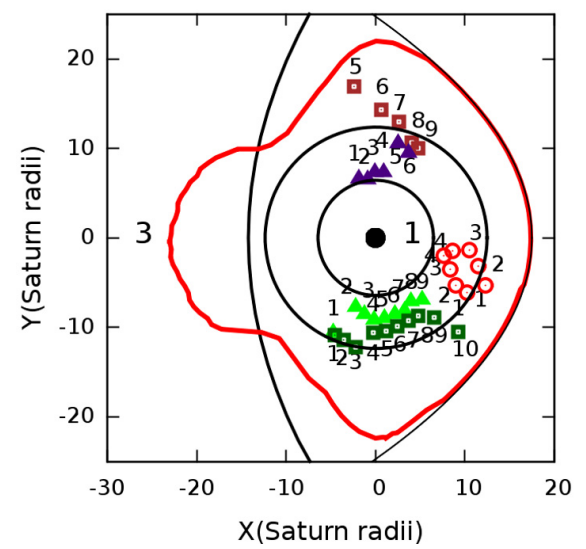

Fig. 11. Southern auroral boundary points for image $C$ mapped into the equatorial plane, in the same format as Fig. 7.

for the dipole field and spherical ionosphere and for the multipole field and spheroidal ionosphere, respectively, the latter again increasing the agreement with the high-latitude auroral edge locations shown by the inner red crosses. Green areas now mark regions with brightness higher that $6 \%$ of a local maximum, defining a dawn arc, near-noon spot, and a dusk patch. The large size of the dusk patch compared with image A may be connected with the positive IMF $B_{y}$ present, opposite to the negative $B_{y}$ present for image A. For fieldline mapping purposes, the boundaries of these auroral regions are marked with symbols in Fig. 10, in the same format as Fig. 6.

Figure 11 shows these points mapped along the model field into the equatorial plane, where the red line divides

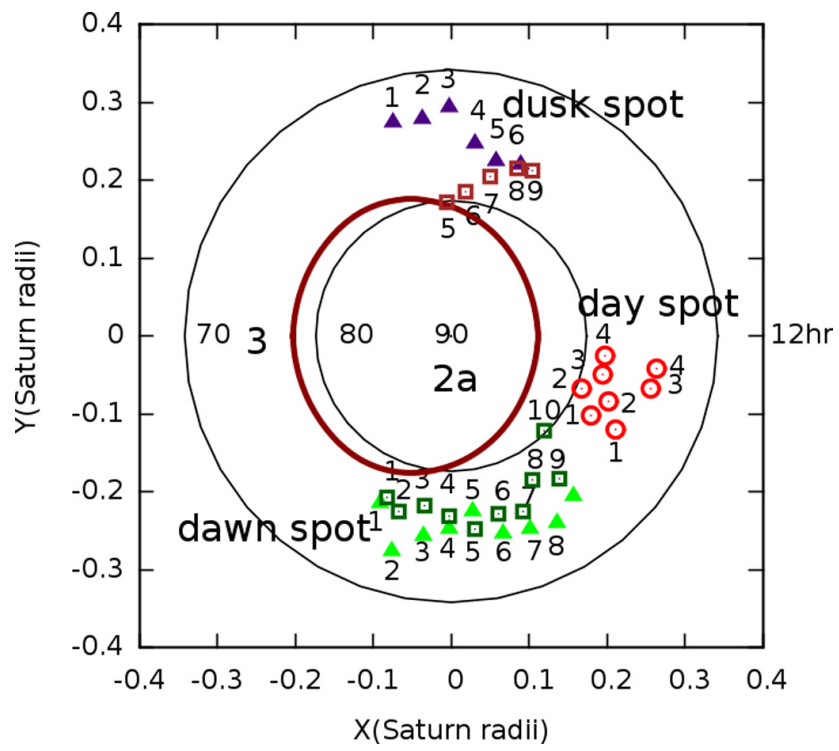

Fig. 12. Southern auroral boundary points for image $C$ mapped into the northern ionosphere, in the same format as Fig. 8.

closed (region 1) and interplanetary field lines (region 3). The dawn arc maps centrally to the dawn ring current, while the near-noon spot maps to the pre-noon ring current. The equatorward boundary of the dusk patch maps to the dusk ring current, while the high-latitude boundary maps between the dusk ring current and the open flux boundary, with points numbered 1-4 being located on open field lines not mapping into the equatorial plane. Overall, these results suggest a connection between these bright auroral features and Saturn's ring current. Figure 12 shows the mapping of these features into the Northern Hemisphere in the same format as Fig. 8. We see that the auroral pattern in the Northern Hemisphere is similar to the original structure in the Southern Hemisphere, except that the shapes of the northern auroral features are narrower in latitude.

\section{Discussion and conclusions}

This paper is devoted to a question of current importance the determination of the origin of Saturn's aurora (see, e.g., Kurth et al., 2009, where the solar wind-magnetosphereionosphere interaction for Saturn is discussed in detail). For this purpose we have mapped bright auroral emission zones observed in one hemisphere into the equatorial magnetosphere, since knowing the location of the projected aurors can lead to better understanding of the physical processes responsible for the emissions. As noted by us earlier (Belenkaya et al., 2011), existing theories of the Kronian auroral generation include inner co-rotation breakdown as discussed previously by Hill (1979) and Cowley and Bunce (2001) for Jupiter, centrifugal interchange (Curtis et al., 1982; Sittler et al., 2006), the KH instability at the magnetopause (Galopeau 
et al., 1995; Masters et al., 2012), and co-rotation breakdown near the open-closed boundary (Cowley and Bunce, 2003; Cowley et al., 2004a, b, 2008). Knowing the regions magnetically conjugate with the auroral zones can clarify which of these mechanisms act in Saturn's magnetosphere and where. In addition, mapping emissions along field lines from one hemisphere to the other will aid interpretation of simultaneous observations of northern and southern auroras.

To these ends we have further analysed two HST UV southern auroral images obtained in 2008, when IMF data were measured simultaneously by the Cassini spacecraft just upstream of the noon bow shock, one under southward and the other under northward IMF conditions. We have firstly compared these images with results obtained using a newly modified version of the paraboloid field model in which the most recently determined multipole planetary field terms are included (specifically axial dipole, quadrupole, and octupole), together with a spheroidal surface representing the planetary ionosphere close to the surface of maximum auroral luminosity. It is found that this model provides a better coincidence between the open flux boundary and the highlatitude auroral oval boundaries than previous models using a dipole field only together with a spherical surface. The decrease in angular radius of the open field boundary due to taking these factors into account is $\sim 3^{\circ}$ latitude in the Northern Hemisphere and $2-2.5^{\circ}$ in the Southern Hemisphere, where the decrease is bigger on the nightside than in the dayside. Inclusion of the quadrupole term also removes the northsouth symmetry in the planetary field, such that the open field region and auroral boundaries lie closer to the pole in the Northern Hemisphere compared with the southern. For image A for southward IMF, for example, we find that the poleward edge of the bright dawn patch is located on average $2.6^{\circ}$ closer to the pole in the Northern Hemisphere than in the Southern Hemisphere, ranging between 0.4 and $4.7^{\circ}$. For the equatorward boundary of this dawn patch the average poleward shift is $3.1^{\circ}$, ranging between 2.0 and $4.6^{\circ}$. However, such mappings cannot be directly tested using the equinoctial images from 2009, because simultaneous IMF data are unfortunately not available.

We have also used the magnetic field model to map the observed southern bright auroral boundaries into the equatorial plane and also into the northern ionosphere. For image A for southward IMF the equatorward boundary of the bright dawn patch maps to the dawn-noon sector of the ring current, while its poleward boundary maps close to the boundary of the open field lines. Emissions in the poleward part of the dawn patch may then be generated by the velocity shears near the open-closed field boundary, while the equatorward part of the emission appears to be generated by field-aligned currents mapping to the dawn ring current. The dusk arc in this image is found to map between the outer edge of ring current and the open field boundary, while the near-noon spot maps to the noon ring current.
For southern image $\mathrm{C}$ obtained during northward IMF, it is shown that the narrow dawn arc maps into the dawn sector of the equatorial ring current, while the near-noon spot maps to the pre-noon ring current. The bright dusk patch in this case maps in the equatorial plane between the open field line boundary and the inner edge of the ring current. The appearance of the large dusk patch in image $C$ may be connected with positive $B_{y}$, opposite to image A for negative $B_{y}$. However, this is a preliminary conclusion, as we have only two examples studied here. The mapping of the dawn arc in this case into the middle of the ring current, unlike in image A where the dawn patch extends close to the boundary of open field lines, suggests that different possibly IMF-dependent mechanisms may be operating in these cases.

It is also shown that for both southward and northward IMF, the projection of the southern bright auroral regions into the Northern Hemisphere gives almost hemispherically symmetric features, although the corresponding northern zones are narrower than their southern counterparts. These results are in overall conformity with the previous results of Nichols et al. (2009) from analysis of simultaneous auroral features observed under near-equinoctial conditions.

Acknowledgements. Work at the Lomonosov Moscow State University, Skobeltsyn Institute of Nuclear Physics (SINP MSU), and the University of Leicester (UoL) was supported by RFBR (grant no. 12-02-92600-a) and London Royal Society (JP080836) Joint Project funding. Work at the SINP MSU was also supported by RFBR grants no. 12-05-00219-a and 11-05-00894-a, and the European FP7 project IMPEx (no. 262863), while work at the UoL was supported by STFC grants ST/H002480/1 and ST/K001000/1. This work employs observations made with the NASA/ESA Hubble Space Telescope, obtained at the Space Telescope Science Institute, which is operated by AURA Inc for NASA.

Topical Editor L. Blomberg thanks two anonymous referees for their help in evaluating this paper.

\section{References}

Alexeev, I. I. and Belenkaya, E. S.: Electric field in an open model of the magnetosphere, Geomagn. Aeron., 23, 57-61, 1983.

Alexeev, I. I. and Belenkaya, E. S.: Modeling of the Jovian Magnetosphere, Ann. Geophys., 23, 809-826, doi:10.5194/angeo-23809-2005, 2005.

Alexeev, I. I., Kalegaev, V. V., Belenkaya, E. S., and Bobrovnikov, S. Y.: Dynamic model of the magnetosphere: Case study for January 9-12, 1997, J. Geophys. Res., 106, 25683-25694, 2001.

Alexeev, I. I., Kalegaev, V. V., Belenkaya, E. S., Bobrovnikov, S. Y., Bunce, E. J., Cowley, S. W. H., and Nichols, J. D.: A global magnetic model of Saturn's magnetosphere, and a comparison with Cassini SOI data, Geophys. Res. Lett., 33, L08101, doi:10.1029/2006GL025896, 2006.

Alexeev, I. I., Belenkaya, E. S., Bobrovnikov, S. Yu., Slavin, J. A., and Sarantos, M.: Paraboloid model of Mercury's magnetosphere, J. Geophys. Res., 113, A12210, doi:10.1029/2008JA013368, 2008. 
Belenkaya, E. S.: Reconnection modes for near-radial IMF, J. Geophys. Res., 103, 41-47, 1998a.

Belenkaya, E. S.: High-latitude ionospheric convection patterns dependent on the variable IMF orientation, J. Atmos. Solar-Terr. Phys., 60, 1343-1354, 1998b.

Belenkaya, E. S., Alexeev, I. I., Kalegaev, V. V., and Blokhina, M. S.: Definition of Saturn's magnetospheric model parameters for the Pioneer 11 flyby, Ann. Geophys., 24, 1145-1156, doi:10.5194/angeo-24-1145-2006, 2006.

Belenkaya, E. S., Alexeev, I. I., Blokhina, M. S., Cowley, S. W. H., Badman, S. V., Kalegaev, V. V., and Grigoryan, M. S.: IMF dependence of the open-closed field line boundary in Saturn's ionosphere, and its relation to the UV auroral oval observed by the Hubble Space Telescope, Ann. Geophys., 25, 1215-1226, doi:10.5194/angeo-25-1215-2007, 2007.

Belenkaya, E. S., Cowley, S. W. H., Badman, S. V., Blokhina, M. S., and Kalegaev, V. V.: Dependence of the open-closed field line boundary in Saturn's ionosphere on both the IMF and solar wind dynamic pressure: comparison with the UV auroral oval observed by the HST, Ann. Geophys., 26, 159-166, doi:10.5194/angeo-26-159-2008, 2008.

Belenkaya, E. S., Alexeev, I. I., Blokhina, M. S., Bunce, E. J., Cowley, S. W. H., Nichols, J. D., Kalegaev, V. V., Petrov, V. G., and Provan, G.: IMF dependence of Saturn's auroras: modelling study of HST and Cassini data from 12-15 February 2008, Ann. Geophys., 28, 1559-1570, doi:10.5194/angeo-281559-2010, 2010.

Belenkaya, E. S., Cowley, S. W. H., Nichols, J. D., Blokhina, M. S., and Kalegaev, V. V.: Magnetospheric mapping of the dayside UV auroral oval at Saturn using simultaneous HST images, Cassini IMF data, and a global magnetic field model, Ann. Geophys., 29, 1233-1246, doi:10.5194/angeo-29-1233-2011, 2011.

Belenkaya, E. S., Alexeev, I. I., Slavin, J. A., and Blokhina, M. S.: Influence of the solar wind magnetic field on the Earth and Mercury magnetospheres in the paraboloidal model, Planet. Space Sci., 75, 46-55, 2013.

Blomberg, L. G., Cumnock, J. A., Alexeev, I. I., Belenkaya, E. S., Bobrovnikov, S. Yu., and Kalegaev, V. V.: Transpolar aurora: time evolution, associated convection patterns, and a possible cause, Ann. Geophys., 23, 1917-1930, doi:10.5194/angeo-231917-2005, 2005.

Burton, M. E., Dougherty, M. K., and Russell, C. T.: Saturn's internal planetary magnetic field, Geophys. Res. Lett., 37, L24105, doi:10.1029/2010GL045148, 2010.

Cowley, S. W. H.: A qualitative study of the reconnection between the Earth's magnetic field and an interplanetary field of arbitrary orientation, Radio Sci., 8, 903-913, 1973.

Cowley, S. W. H. and Bunce, E. J.: Origin of the main auroral oval in Jupiter's coupled magnetosphere-ionosphere system, Planet. Space Sci., 49, 1067-1088, 2001.

Cowley, S. W. H. and Bunce, E. J.: Corotation-driven magnetosphere-ionosphere coupling currents in Saturn's magnetosphere and their relation to the auroras, Ann. Geophys., 21, 1691-1707, doi:10.5194/angeo-21-1691-2003, 2003.

Cowley, S. W. H. and Hughes, W. J.: Observation of an IMF sector effect in the $Y$ magnetic field component at geostationary orbit, Planet. Space Sci., 31, 73-90, 1983.
Cowley, S. W. H., Bunce, E. J., and O'Rourke, J. M.: A simple quantitative model of plasma flows and currents in Saturn's polar ionosphere, J. Geophys. Res., 109, A05212, doi:10.1029/2003JA010375, 2004a.

Cowley, S. W. H., Bunce, E. J., and Prangé, R.: Saturn's polar ionospheric flows and their relation to the main auroral oval, Ann. Geophys., 22, 1379-1394, doi:10.5194/angeo-22-13792004, 2004b.

Cowley, S. W. H., Arridge, C. S., Bunce, E. J., Clarke, J. T., Coates, A. J., Dougherty, M. K., Gérard, J.-C., Grodent, D., Nichols, J. D., and Talboys, D. L.: Auroral current systems in Saturn's magnetosphere: comparison of theoretical models with Cassini and HST observations, Ann. Geophys., 26, 2613-2630, doi:10.5194/angeo-26-2613-2008, 2008.

Curtis, S. A., Lepping, R. P., and Sittler, E. C.: The centrifugal flute instability and the generation of Saturnian kilometric radiation, J. Geophys. Res., 91, 10989-10994, 1982.

Dungey, J. W.: Interplanetary magnetic field and the auroral zones, Phys. Rev. Lett., 6, 47-48, 1961.

Galopeau, P., Zarka, P., and Lequéau, D.: Source location of Saturn's kilometric radiation: The Kelvin-Helmholtz instability hypothesis, J. Geophys. Res., 100, 26397-26410, 1995.

Gérard, J.-C., Grodent, D., Gustin, J., and Saglam, A.: Characteristics of Saturn's FUV aurora observed with the Space Telescope Imaging Spectrograph, J. Geophys. Res., 109, A09207, doi:10.1029/2004JA010513, 2004.

Gérard, J.-C., Bonfond, B., Gustin, J., Grodent, D., Clarke, J. T., Bisikalo, D., and Shematovich, V.: Altitude of Saturn's aurora and its implication for the characteristic energy of precipitated electrons, Geophys. Res. Lett., 36, L02202, doi:10.1029/2008GL036554, 2009.

Hill, T. W.: Inertial limit on corotation, J. Geophys. Res., 84, 6554 6558, 1979.

Kurth, W. S., Bunce, E. J., Clarke, J. T., Crary, F. J., Grodent, D. G., Ingersoll, A. P., Dyudina, U. A., Lamy, L., Mitchell, D. G., Persoon, A. M., Pryor, W. R., Saur, J., and Stallard, T.: Auroral processes, in: Saturn from Cassini-Huygens, edited by: Dougherty, M. K., Esposito, L.W., and Krimigis, S. M., Springer Science \& Business Media, pp. 333-374, doi:10.1007/978-1-4020-92176_12, 2009.

Masters, A., Achilleos, N., Cutler, J. C., Coates, A. J., Dougherty, M. K., and Jones, G. H.: Surface waves on Saturn's magnetopause, Planet. Space Sci., 65, 109-121, 2012.

Meredith, C. J., Cowley, S. W. H., Hansen, K. C., Nichols, J. D., and Yeoman, T. K.: Simultaneous conjugate observations of smallscale structures in Saturn's dayside ultraviolet auroras - implications for physical origins, J. Geophys. Res., 118, 2244-2266, doi:10.1002/jgra.50270, 2013.

Nichols, J. D., Badman, S. V., Bunce, E. J., Clarke, J. T., Cowley, S. W. H., Crary, F. J., Dougherty, M. K., Gérard, J.-C., Grodent, D., Hansen, K. C., Kurth, W. S., Mitchell, D. G., Pryor, W. R., Stallard, T. S., Tallboys, D. L., and Wannawichian, S.: Saturn's equinoctial auroras, Geophys. Res. Lett., 36, L24102, doi:10.1029/2009GL041491, 2009.

Petrukovich, A. A.: Origins of plasma sheet $B_{y}$, J. Geophys. Res., 116, A07217, doi:10.1029/2010JA016386, 2011. 
Radioti, A., Grodent, D., Gérard, J.-C., Milan, S. E., Bonfond, B., Gustin, J., and Pryor, W.: Bifurcations of the main auroral ring at Saturn: ionospheric signatures of consecutive reconnection events at the magnetopause, J. Geophys. Res., 116, A11209, doi:10.1029/2011JA016661, 2011.
Sittler, E. C., Blanc, M. F., and Richardson, J. D.: Proposed model for Saturn's auroral response to the solar wind: Centrifugal instability model, J. Geophys. Res., 111, A06208, doi:10.1029/2005JA011191, 2006. 